VII.

Aus der mediz- Klinik zu Sraßburg.

(Dir. Prof. Dr. Moritz.)

\title{
Über den Abbau von Fettsäuren beim Diabetes melitus.
}

(IV. Mitteilung.)

Von

Dr. Julius Baer und Dr. Leon Blum.

(Mit 6 Kurven.)

In früheren Mitteilungen 1) stellten wir tuber die Bildung der $\beta$-Oxybuttersäure beim mensehlichen Diabetes neben anderen folgende Tatsachen fest. Darreichung

1. von Buttersäure u. Capronsăure

2. von $\beta$-Methylbuttersäure (Isovaleriansäure) und $\beta$-Aethy\}buttersäure

3. der Aminosäuren Leucin, Phenylalanin u. Tyrosin bewirkt eine Vermehrung der ausgeschiedenen Acidosekörper.

Wir bezogen diese Vermebrung auf einen nach bestimmten chemischen Regeln sich vollziebenden Übergang der normalen und in $\beta$-Stellung verzweigten Fettsäuren in Oxybuttersäure ${ }^{2}$ ) Für die Aminosäuren mußten wir hierbei einen intermediären Übergang in Säuren einer dieser beiden Gruppen annebmen (auch bei den aromatischen Aminosäuren). Wie wir hervorboben, erschien uns Wiederholung einzelner Versuche wünsehenswert, die uns damals infolge des Mangels an geeigneten Patienten nicht möglich war. Wir haben daher diese Untersuchungen bei Gelegenheit noch zu ergänzen gesucht, besonders da wir eine Übertragung der Resultate, die Embden bei Leberdurchblutungen, z. T. mit den gleichen Substanzen gewonnen hat, auf den menseblichen Stoffwechsel nicht obne weiteres für statthaft hielten. (Vgl. hiertiber auch Ma g n u s-Lev y 3).

1) Dies Archiv Bd. 55, Bd. 56 (1906) und Bd. 59 (1908).

2) Über den chemischen Prozeß beim Utbergang der in $a$ - Stellung substituierten Fettsäuren, für die $\alpha$-Methylbuttersäure und scheinbar auch für die $\alpha-$ Äthylbuttersäure gaben unsere Versuche keine genügende Erklärung.

3) Magnus-Levy. Ergebnisse der inneren Medizin. Bd. I. 
Bei diesen Versuchen ergaben sich noch eine Anzabl von Fragestellungen, auf die wir bei der Besprechung der Resultate eingehen werden.

Die Versuche wurden in gleicher Weise wie die fruheren angestellt: Darreichung einer stets gleich bleibenden Kost und derselben täglichen Menge von Natron bicarbonicum. Die Substanzen wurden stets mit den berechneten Mengen von Natron bicarbonicum verabreicht.

Der Diabetiker, an dem wir die folgenden Untersuchungen anstellten, war derselbe Patient, an dem wir die Versuche I u. II der 3. Mitteilung vorgenommen hatten.

Wie aus den Tabellen hervorgeht, hatte die Krankheit sich inzwischen wesentlich versehlimmert; die Acidose hatte erheblich zugenommen, im Versuch I zeigt sie mittlere Intensität, im Versuch II erreicht sie eine erhebliche Stärke.

Tabelle I.

\begin{tabular}{|c|c|c|c|c|c|c|c|c|c|}
\hline 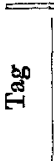 & 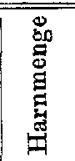 & 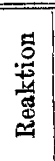 & 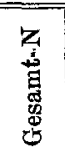 & 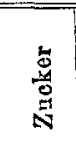 & 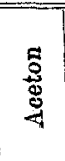 & 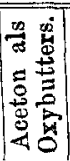 & 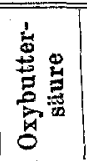 & 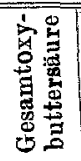 & Dargereichte Substanz \\
\hline $\begin{array}{l}1 . \\
2 . \\
3 . \\
4 . \\
5 .\end{array}$ & $\begin{array}{l}1700 \\
1900 \\
1350 \\
1850 \\
1750\end{array}$ & $\begin{array}{l}\text { alk. } \\
= \\
= \\
= \\
=\end{array}$ & $\begin{array}{l}12.7 \\
14.5 \\
14.6 \\
14.0 \\
13.3\end{array}$ & $\begin{array}{l}34.0 \\
42 \\
27 \\
30.75 \\
23\end{array}$ & $\begin{array}{l}2.73 \\
2.61 \\
2.01 \\
2.56 \\
3.35\end{array}$ & $\begin{array}{l}4.89 \\
4.57 \\
3.52 \\
4.48 \\
5.87\end{array}$ & $\begin{array}{l}11.54 \\
15.02 \\
13.13 \\
12.01 \\
19.22\end{array}$ & $\begin{array}{l}16.43 \\
17.59 \\
16.65 \\
16.49 \\
25.09\end{array}$ & $26 \mathrm{~g}$ synthet. d-1. Leucin \\
\hline $\begin{array}{l}6 . \\
7 . \\
8 . \\
9 .\end{array}$ & $\begin{array}{l}1800 \\
2100 \\
2000 \\
2200\end{array}$ & $\begin{array}{l}= \\
= \\
=\end{array}$ & $\begin{array}{l}15.1 \\
14.1 \\
12.5 \\
14.2\end{array}$ & $\begin{array}{l}34 \\
31 \\
34 \\
35\end{array}$ & $\begin{array}{l}2.54 \\
2.64 \\
2.17 \\
4.46\end{array}$ & $\begin{array}{l}4.45 \\
4.63 \\
3.19 \\
7.82\end{array}$ & $\begin{array}{r}14.48 \\
13.19 \\
9.30 \\
13.69\end{array}$ & $\begin{array}{l}18.93 \\
17.82 \\
13.09 \\
21.51\end{array}$ & $33 \mathrm{~g}$ synthet. Phenylalanin \\
\hline $\begin{array}{l}10 . \\
11 . \\
12 . \\
13 .\end{array}$ & $\begin{array}{l}2200 \\
2400 \\
2100 \\
2300\end{array}$ & $\begin{array}{l}= \\
= \\
= \\
=\end{array}$ & $\begin{array}{l}14.1 \\
13.1 \\
14.2 \\
14.0\end{array}$ & $\begin{array}{l}36 \\
29 \\
33 \\
41\end{array}$ & $\begin{array}{l}2.43 \\
2.12 \\
3.13 \\
3.96\end{array}$ & $\begin{array}{l}4.25 \\
5.68 \\
5.48 \\
6.94\end{array}$ & $\begin{array}{l}11.24 \\
13.42 \\
16.59 \\
23.21\end{array}$ & $\begin{array}{l}15.49 \\
17.10 \\
22.07 \\
30.15\end{array}$ & $17.6 \mathrm{~g}$ Buttersäure \\
\hline $\begin{array}{l}14 . \\
15 .\end{array}$ & $\begin{array}{l}1500 \\
1700\end{array}$ & $\begin{array}{l}= \\
=\end{array}$ & $\begin{array}{l}12.7 \\
12.8\end{array}$ & $\begin{array}{l}56 \\
46\end{array}$ & $\begin{array}{l}2.68 \\
2.42\end{array}$ & $\begin{array}{l}4.10 \\
4.23\end{array}$ & $\begin{array}{l}15.35 \\
12.01\end{array}$ & $\begin{array}{l}20.05 \\
16.24\end{array}$ & \\
\hline
\end{tabular}

Kost: $300 \mathrm{~g}$ Rahm, 3 Eier, $100 \mathrm{~g}$ Fleisch, $950 \mathrm{~g}$ Gemuse, $50 \mathrm{~g}$ Speck, $50 \mathrm{~g}$ Butter $500 \mathrm{~g}$ Wein, $20 \mathrm{~g} \mathrm{Na}$ bicarbonicum.

Im Versuch I (Juli 1908) erhielt Pat. am 5. Tage $26 \mathrm{~g}$ synthetisches nach E. Fischer bergestelltes Leucin. Die Oxybuttersäureausscheidung stieg um 8,4 g, (davon entfallen auf Aceton $1,4 \mathrm{~g}$ als Oxybuttersäure berechnet); am Nachtage betrug die Vermehrung noch $2 \mathrm{~g}$ über die an den Vortagen ausgeschiedenen Mengen. Statt der theoretisch möglichen Menge von $20,8 \mathrm{~g}$ entstanden demnach ca $10,4 \mathrm{~g}$, also etwa 50 Proz.; es ist dies zufällig derselbe Wert, den wir auch in unserem früheren Versuche (Mitteil. I.) mit Eiweißleucin erhalten hatten. 
Kurve 1 (aus Mitteilung 1).
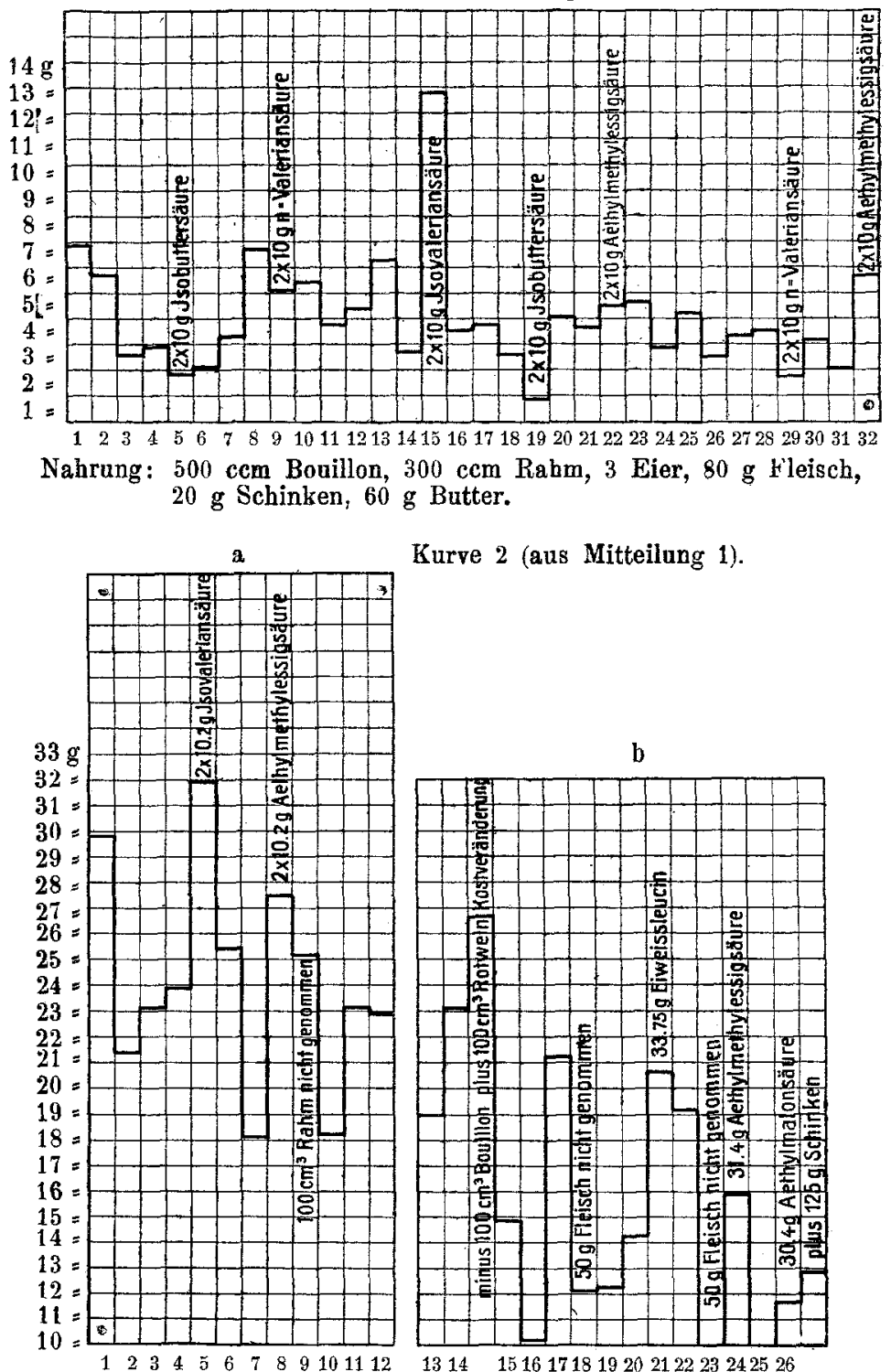

Kurve 2 (aus Mitteilung 1).

Nahrung: $400 \mathrm{ccm}$ Rahm,

40 g Speck.

$200 \mathrm{~g}$ Fleisch,

$300 \mathrm{~g}$ Gemüse,

$600 \mathrm{ccm}$ Bouillon,

$80 \mathrm{~g}$ Butter,

4 Eier,

$100 \mathrm{ccm}$ Rotwein.

b

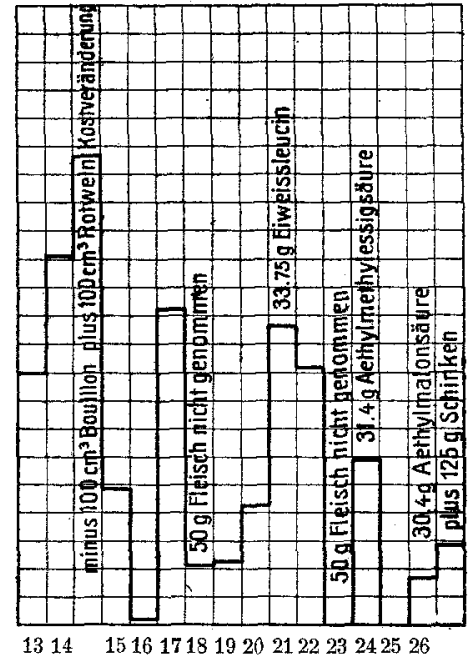

bo Nahrung: $300 \mathrm{ccm}$ Rahm,

$40 \mathrm{~g}$ Speck,

$200 \mathrm{~g}$ Fleisch,

$300 \mathrm{~g}$ Gemüse,

$600 \mathrm{ccm}$ Bouillon,

60 g Butter,

4 Eier,

$300 \mathrm{ccm}$ Rotwein. 
Am 11. Tage wurden $33 \mathrm{~g}$ synthetisch nach Erlenmeyer hergestelltes Phenylalanin verabreicht. Die Mehrausseheidung an Oxybuttersäure betrug dem Vortag gegenuber $8,4 \mathrm{~g}$ (davon $4,1 \mathrm{~g}$ auf Aceton, als Oxybuttersäure gerechnet). An den beiden Nachtagen war die Ausscheidung wieder annähernd normal. Am 14. Tage stieg die Oxybuttersäureausscheidung an, gleichzeitig wurde auch die Zuckerausscheidung inkonstant. In diese etwas unregelmäßige Periode fällt die Darreichung von Buttersäure, die einen Anstieg der Oxybuttersäureansseheidung bewirkt. Doch ist der Versuch wegen dieser Schwankung nicht, wie wir es beabsichtigt hatten, zum Vergleich mit den Leucin- und Phenylalaninversuchen verwertbar. Auffällig erscheint an diesem, wie in unserem ersten Versuche ${ }^{1)}$ mit Phenylalanin die starke Vermehrung der Acetonausscheidung im Vergleich zur Ausscheidung der Oxybuttersäure.

Der Versuch II wurde 7 Monate später (Februar-März 1909) ausgeführt. Nachdem Patient Juli 1908 zuckerfrei (nach Gemüsetag und Glutarsäure darreichung) entlassen war, beging er zu Hause Exzesse im Genuß von Kohlehydraten, die er in größeren Mengen (Brot, Kartoffeln, Trauben) zu sich nahm. Er kam dann im Zustande beginnenden Comas bereits mit großer Atmung in die Klinik. In dieser Periode der Säurevergiftung schied Patient in den ersten 3 Tagen $365 \mathrm{~g}$ Oxybuttersäure (incl. Aceton) im Urin nach Zufuhr von $340 \mathrm{~g}$ Natron bicarbonicum aus. 14 Tage darauf wurde mit dem Versuche begonnen, als alle Zeichen der Intoxikation versehwunden waren und der Harn nach täglicher Eingabe von $50 \mathrm{~g}$ Natron bicarbonicum alkalische Reaktion aufwies. In dieser Versuchsreihe liegen die Oxybuttersäurewerte viel höher, im Mittel etwa $40 \mathrm{~g}$, die Ausscheidung ist außerdem unregelmäßig. Nur in einer Zwischenperiode (vom 14.-24. oder 26. Tage) sind die täglichen Werte ziemlich konstant, sodaß wir wenigstens für diesen Zeitraum die Resultate als brauchbar ansehen können. Glücklicherweise fielen gerade in diese Periode die von uns bereits früher (Vgl. Mitteil. III.) beabsichtigten Versuche mit den beiden optisch aktiven Leucinen. Die Substanzen waren nach dem Verfahren von E. Fischer ${ }^{2}$ ) hergestellt, das d-Leucin zeigte in 5 prozentiger Lösung: in 20 Proz. Salzsäure eine Drehung von $\alpha \mathrm{D}:-15.3$, das l-Leucin von $\alpha \mathrm{D}=+15.1^{\circ}$ (nach E. Fischer $+15,6^{\circ}$ ).

Das d-Leucin brachte am 16 . Versuchstage nur eine geringe Vermehrung der Oxybuttersäure hervor, 2,9 g, eine Abweichung, die noch in die täglichen Schwankungen fällt.

1) 1. Mitteilung 1. c.

2) E. Fischer u. War rourg. Ber. d. Deuts. Chem. Gesellsch. XXXIX 3997. 
Das mit dem Eiweißleucin identische 1 Leucin brachte eine erhebliche Vermehrung der Oxybuttersäureausscheidung zustande: 10,6 g, (davon $4 \mathrm{~g}$ Aceton, als Oxybuttersäure berechnet); am Nachtag betrug die Vermehrung noch etwa $6,4 \mathrm{~g}$ (davon $0,4 \mathrm{~g}$ auf Aceton, als 0xybuttersäure berechnet.)

Leider begannen nach dieser Zeit die Oxybuttersäurewerte unregelmäßig zu werden, sodaß der Versuch mit dem 1-Leucin weniger eindeutig erscheint wie der mit d-Lencin. Jedenfalls ist die Vermehrung: im Gegensatz zum d-Leucin recht beträchtlich, sie stimmt außerdem

Kurve 3 (aus Mitteilung 2).

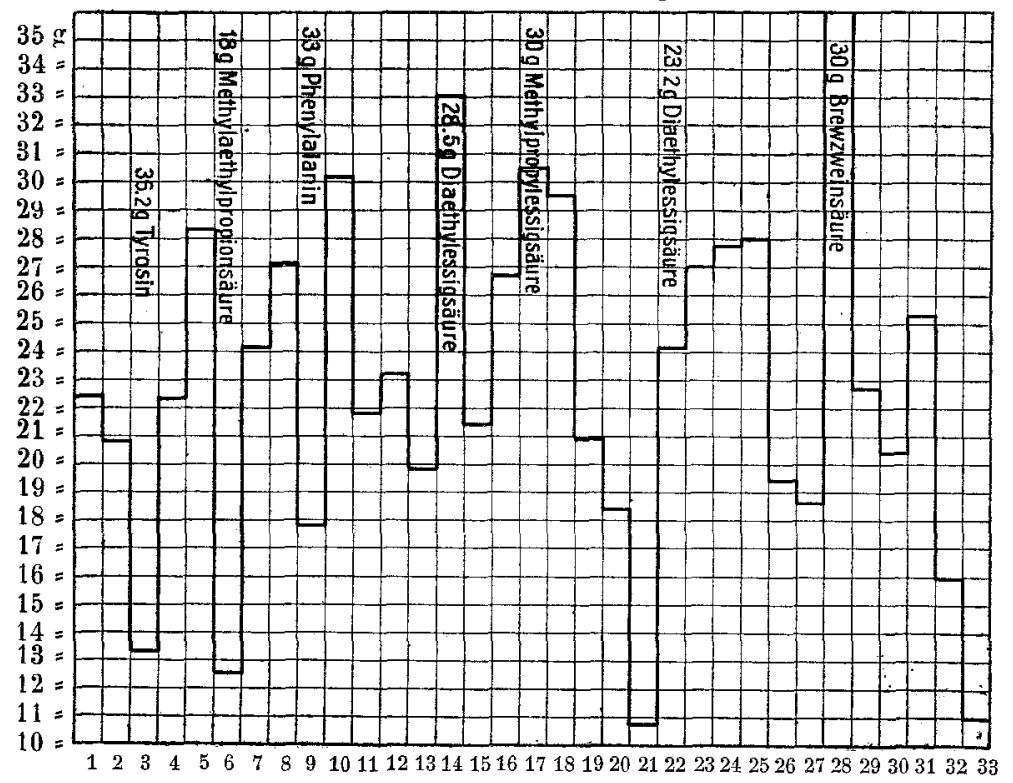

Kost: $200 \mathrm{ccm}$ Rahm, $200 \mathrm{~g}$ Fleisch, $100 \mathrm{~g}$ Schinken, $200 \mathrm{~g}$ Sauerkraut, $20 \mathrm{~g}$ Butter, $500 \mathrm{ccm}$ Wein, $600 \mathrm{ccm}$ Bouillon, $40 \mathrm{~g} \mathrm{NaHCO}_{3}$.

mit den Resultaten unserer früheren Versuche mit Eiweißleuein IMitt. I) und synthetischem Leucin (Tabelle I dieser Arbeit) überein.

Die übrigen Versuche mit Buttersäure (Tag 7 u. 48) Isovaleriansäure (Tag 11 u. 43) Phenylalanin (Tag 32) Tyrosin (27. Tag) und (socapronsäure (Tag 35) sind wegen der großen Schwankungen unbrauchbar. Die Ursachen dieser Schwankungen waren meist nicht zu erkennen, nur für die am 33. Tage aufgetretene Vermehrung. der Oxybuttersäureausseheidung ist wohl eine leichte Erkältung mit geringem Fieber verantwortlich zu machen. Immerhin balten wir es für angebracht, die Kurve der ganzen Versuchsreihe wiederzu- 
Tabelle II.

\begin{tabular}{|c|c|c|c|c|c|c|c|c|c|c|}
\hline$\stackrel{\infty}{\leftarrow \infty}$ & 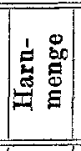 & 몽 & 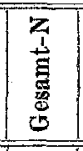 & 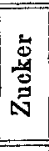 & $\frac{\Xi}{5}$ & $\mid \begin{array}{l}0 \\
0 \\
0\end{array}$ & 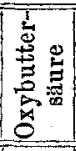 & 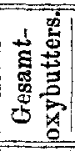 & Dargereichte Substanz & $\begin{array}{l}\text { Bemer- } \\
\text { kungen }\end{array}$ \\
\hline $\begin{array}{l}1 . \\
2 . \\
3 . \\
4 . \\
5 . \\
6 . \\
7 .\end{array}$ & $\cdot\left|\begin{array}{l}3200 \\
3000 \\
2700 \\
2800 \\
2750 \\
3150 \\
2600\end{array}\right|$ & $\begin{array}{l}\text { alk. } \\
= \\
= \\
= \\
= \\
=\end{array}$ & $\mid \begin{array}{r}- \\
- \\
12.66 \\
11.45 \\
13.12 \\
12.79 \\
9.54\end{array}$ & & $\begin{array}{l}7.05 \\
6.15 \\
5.95 \\
6.34 \\
5.53 \\
6.58 \\
6.39\end{array}$ & $\begin{array}{r}12.65 \\
11.03 \\
10.67 \\
11.36 \\
9.92 \\
11.80 \\
11.45\end{array}$ & $\begin{array}{l}- \\
- \\
40.64 \\
40.86 \\
38.98 \\
47.08 \\
45.28\end{array}$ & $\begin{array}{l}-\overline{ } \\
51.31 \\
52.22 \\
48.90 \\
59.88 \\
56.73\end{array}$ & Buttersäure $17.6+16.8 \mathrm{~g} \mathrm{NaHCO}_{3}$ & \\
\hline $\begin{array}{l}8 . \\
9 . \\
10 . \\
11 .\end{array}$ & $\left|\begin{array}{l}3600 \\
2450 \\
3300 \\
3050\end{array}\right|$ & $\begin{array}{l}= \\
= \\
=\end{array}$ & $\begin{array}{l}10.68 \\
11.6 \\
12.84 \\
13.79\end{array}$ & & $\begin{array}{l}5.94 \\
5.07 \\
5.74 \\
8.02\end{array}$ & $\begin{array}{c}10.67 \\
9.09 \\
10.3 \\
14.38\end{array}$ & \begin{tabular}{|}
37.38 \\
29.70 \\
34.82 \\
42.92
\end{tabular} & $\left|\begin{array}{c}48.05 \\
38 \cdot 79 \\
45.12 \\
57.4\end{array}\right|$ & Isovalerians. $20.4+16.8 \mathrm{~g} \mathrm{NaHCO}_{3}$ & \\
\hline $\begin{array}{l}12 . \\
13 . \\
14 . \\
15 . \\
16 .\end{array}$ & $\left|\begin{array}{l}3300 \\
3000 \\
3100 \\
3800 \\
3450\end{array}\right|$ & $\begin{array}{l}= \\
= \\
= \\
=\end{array}$ & \begin{tabular}{|l|}
12.94 \\
11.47 \\
12.84 \\
12.73 \\
15.17
\end{tabular} & & $\begin{array}{l}4.88 \\
5.69 \\
4.74 \\
5.88 \\
5.27\end{array}$ & $\begin{array}{r}8.77 \\
10.2 \\
8.49 \\
10.54 \\
9.45\end{array}$ & $\begin{array}{l}26.12 \\
33.64 \\
23.38 \\
25.06 \\
29.04\end{array}$ & 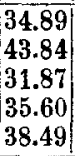 & $26 \mathrm{~g}$ d-Leucin $+16.8 \mathrm{~g} \mathrm{NaHCO}$ & \\
\hline $\begin{array}{l}17 . \\
18 . \\
19 . \\
20 . \\
21 . \\
22 .\end{array}$ & $\left|\begin{array}{l}2300 \\
2500 \\
2850 \\
2600 \\
5050 \\
3500\end{array}\right|$ & $\begin{array}{l}= \\
= \\
= \\
= \\
=\end{array}$ & $\begin{array}{l}12.30 \\
10.34 \\
12.85 \\
13.46 \\
12.75 \\
13.08\end{array}$ & & $\begin{array}{l}4.67 \\
4.79 \\
5.02 \\
4.02 \\
5.22 \\
7.48\end{array}$ & $\begin{array}{r}8.37 \\
8.58 \\
8.99 \\
7.21 \\
9.36 \\
13.41\end{array}$ & \begin{tabular}{|l|}
26.03 \\
24.93 \\
26.47 \\
23.78 \\
29.59 \\
36.20 \\
\end{tabular} & $\begin{array}{l}34.40 \\
33.51 \\
35.46 \\
30.99 \\
38.95 \\
49.61\end{array}$ & $26 \mathrm{~g}$ 1-Leucin $+16.8 \mathrm{~g} \mathrm{NaHCO}$ & \\
\hline $\begin{array}{l}23 . \\
24 . \\
25 . \\
26 . \\
27 .\end{array}$ & $\begin{array}{l}2800 \\
2850 \\
2900 \\
3200 \\
3050\end{array}$ & $\begin{array}{l}= \\
= \\
= \\
=\end{array}$ & $\mid \begin{array}{l}13.05 \\
12.01 \\
11.69 \\
12.86 \\
12.82\end{array}$ & $\begin{array}{l}61 \\
57 \\
50 \\
58 \\
61\end{array}$ & \begin{tabular}{|l|}
5.69 \\
5.46 \\
4.65 \\
5.51 \\
6.017 \\
\end{tabular} & $\begin{array}{r}10.20 \\
9.78 \\
8.35 \\
9.88 \\
10.79\end{array}$ & $\mid \begin{array}{l}35.06 \\
29.13 \\
37.39 \\
33.9 \\
34.65\end{array}$ & \begin{tabular}{|l|}
45.26 \\
38.91 \\
45.74 \\
43.78 \\
45.44
\end{tabular} & $34 \mathrm{~g}$ Tyrosin $+16.8 \mathrm{~g} \mathrm{NaHCO}_{3}$ & \\
\hline $\begin{array}{l}28 . \\
29 . \\
30 . \\
31 . \\
32 .\end{array}$ & $\begin{array}{l}2900 \\
3200 \\
3050 \\
2900 \\
3600\end{array}$ & $\begin{array}{l}= \\
= \\
= \\
=\end{array}$ & $\begin{array}{l}10.39 \\
12.27 \\
12.73 \\
13.89 \\
14.01\end{array}$ & & \begin{tabular}{|l|}
5.01 \\
4.33 \\
4.07 \\
4.32 \\
6.93
\end{tabular} & $\begin{array}{r}8.99 \\
7.77 \\
7.30 \\
7.75 \\
12.42\end{array}$ & $\left|\begin{array}{l}37.33 \\
2295 \\
17.58 \\
25.31 \\
32.24\end{array}\right|$ & $\mid \begin{array}{l}46.32 \\
30.72 \\
24.88 \\
33.06 \\
44.66\end{array}$ & $33 \mathrm{~g}$ Phenylalanin $+16.8 \mathrm{~g} \mathrm{NaHCO}_{3}$ & \\
\hline $\begin{array}{l}33 . \\
34 . \\
35 .\end{array}$ &.$\left|\begin{array}{l}3050 \\
2950 \\
2900\end{array}\right|$ & $\begin{array}{l}= \\
=\end{array}$ & $\mid \begin{array}{l}14.26 \\
13.83 \\
12.54\end{array}$ & $\begin{array}{l}74 \\
71 \\
51\end{array}$ & $\begin{array}{l}7.67 \\
6.504 \\
8.16\end{array}$ & $\begin{array}{l}13.75 \\
11.66 \\
14.63\end{array}$ & $\begin{array}{l}41.73 \\
34.61 \\
32.38\end{array}$ & $\mid \begin{array}{l}55.48 \\
46.27 \\
47.01\end{array}$ & 22.8 Isocaprons. $+16.8 \mathrm{~g} \mathrm{NaHCO}_{3}$ & $\begin{array}{l}\text { Fieber, } \\
\text { Kopf= } \\
\text { schmerzen }\end{array}$ \\
\hline $\begin{array}{l}36 . \\
37 . \\
38 . \\
39 . \\
40 . \\
41 . \\
42 . \\
43 . \\
44 .\end{array}$ & $\cdot \begin{array}{l}3600 \\
3600 \\
2800 \\
2850 \\
3000 \\
3150 \\
2800 \\
2850 \\
3350\end{array}$ & $\begin{array}{l}= \\
= \\
= \\
= \\
= \\
= \\
=\end{array}$ & \begin{tabular}{|l|}
13.18 \\
13.5 \\
16.11 \\
12.32 \\
12.96 \\
13.41 \\
12.00 \\
13.85 \\
12.22
\end{tabular} & $\begin{array}{l}65 \\
80 \\
79 \\
66 \\
60 \\
82 \\
67 \\
67 \\
67\end{array}$ & $\begin{array}{l}8.36 \\
8.84 \\
6.61 \\
7.72 \\
7.72 \\
8.28 \\
7.15 \\
8.38 \\
9.01\end{array}$ & $\begin{array}{l}14.98 \\
15.86 \\
11.85 \\
13.84 \\
13.84 \\
14.86 \\
12.82 \\
15.02 \\
16.15\end{array}$ & $\begin{array}{l}44.18 \\
46.49 \\
31.11 \\
38.7 \\
41.6 \\
41.33 \\
42.18 \\
44.84 \\
49.58\end{array}$ & \begin{tabular}{|l|}
59.16 \\
61.35 \\
42.96 \\
52.54 \\
55.44 \\
56.19 \\
55.50 \\
59.86 \\
65.73
\end{tabular} & $20.4 \mathrm{~g}$ Isovalerians. $+16.8 \mathrm{~g} \mathrm{NaHCO}_{3}$ & \\
\hline $\begin{array}{l}45 . \\
46 . \\
47 . \\
48 .\end{array}$ & $\left|\begin{array}{l}3100 \\
3600 \\
3350 \\
3450\end{array}\right|$ & $\begin{array}{l}= \\
= \\
=\end{array}$ & $\mid \begin{array}{l}11.86 \\
13.41 \\
12.15 \\
12.90\end{array}$ & $\begin{array}{r}65 \\
70 \\
69 \\
100\end{array}$ & $\begin{array}{l}7.37 \\
8.22 \\
7.97 \\
8.27\end{array}$ & $\begin{array}{l}13.21 \\
14.73 \\
14.29 \\
14.84\end{array}$ & $\begin{array}{l}43.18 \\
49.10 \\
47.29 \\
40.40\end{array}$ & \begin{tabular}{|l|}
56.39 \\
63.83 \\
61.58 \\
55.25
\end{tabular} & $17.6 \mathrm{~g}$ Butters. $+16.8 \mathrm{~g} \mathrm{NaHCO} 3$ & \\
\hline $\begin{array}{l}49 . \\
50 .\end{array}$ &.$\left|\begin{array}{l}2700 \\
2400\end{array}\right|$ & $=$ & $\mid \begin{array}{l}13.23 \\
12.20\end{array}$ & & $\begin{array}{l}7 . \overline{15} \\
4.41\end{array}$ & $\begin{array}{r}12.83 \\
7.91\end{array}$ & $\begin{array}{l}38.95 \\
20.6\end{array}$ & $\begin{array}{l}51.78 \\
28.51\end{array}$ & & \\
\hline
\end{tabular}

Kost: $300 \mathrm{~g}$ Rahm, 4 Eier, $100 \mathrm{~g}$ Fleisch, $1000 \mathrm{~g}$ Gemuse, $40 \mathrm{~g}$ Butter, 1 Apfel, $500 \mathrm{~g}$ Wein, $50 \mathrm{~g} \mathrm{Na}$ bicarbonicum. 
geben, da sie nicht allein zur Beurteilung der Versuche nötig ist, sondern weil sie einen Beitrag zur Oxybuttersăureausscheidung im schweren Diabetes bringt. So zeigt die Kurve deutlich, wie bei Absinken der Acidose (Tag 7 und 48) trotz starker Acidose selbst die Darreichung von Buttersäure ohne irgendwelche Wirkung ist.

Etwas Ähnliches findet sich am 27. Tag, an dem offenbar in einer Periode beginnenden Absinkens der Oxybuttersäureausscheidung

Kurven 4, 5 und 6 betreffen denselben Patienten zu verschiedenen Zeiten (s. S. 130).

Kurve 4 (ans Mitteilung 3).

Nov. 1907.

Kost: $300 \mathrm{~g}$ Rahm,

20 g Speck,

3 Eier,

$200 \mathrm{~g}$ Fleisch,

$800 \mathrm{~g}$ Gemüse,

$40 \mathrm{~g}$ Butter,

$250 \mathrm{ccm}$ Wein.

$25 \mathrm{~g} \mathrm{NaHCO}_{3}$.

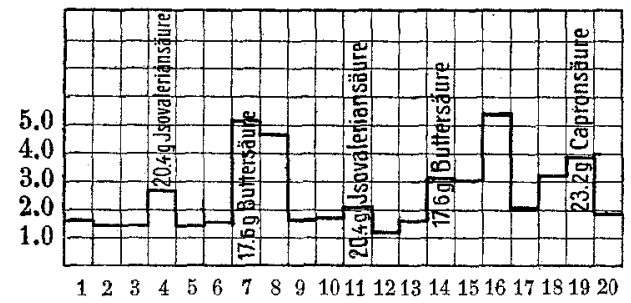

Kurve 5 (Tabelle I dieser Arbeit).

Juli 1908.

Kost: $300 \mathrm{ccm}$ Rahm, $100 \mathrm{~g}$ Fleisch, 3 Eier, $50 \mathrm{~g}$ Speck, $250 \mathrm{~g}$ Gemüse, $50 \mathrm{~g}$ Butter, $500 \mathrm{~g}$ Wein, $20 \mathrm{~g} \mathrm{NaHCO}_{3}$.

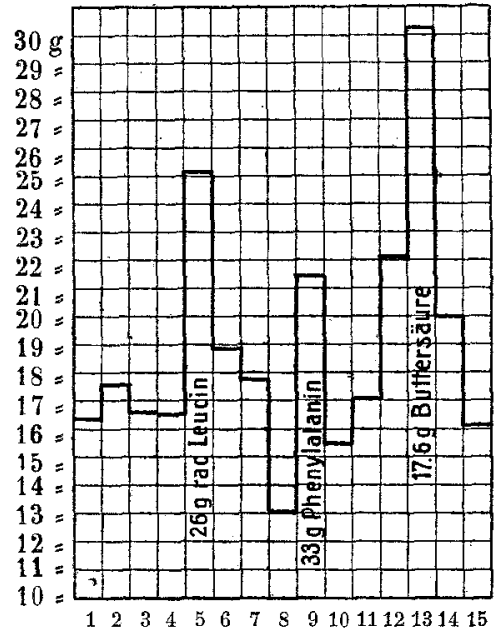

Tyrosin gegeben wurde; dieses beeinflußte scheinbar die Oxybuttersäureansscheidung gar nicht.

Die Versuche bestätigen somit unsere früheren Resultate tuber die Oxybuttersäurebildung aus Phenylalanin und 1-Leucin. D-Leucin 
war gegen unsere Erwartungen viel weniger oder nicht wirksam. Man muß daher annehmen, daß die sterische Konfiguration der Amidosäure, die bei der Bildung der Oxybuttersäure verschwindet, doch für den Abbau des Leueins von Bedeutung ist: lieferten nämlich beide Leucine in gleicher Weise Isovaleriansäure, so ist der Untersohied in ihrer Wirkung auf die Oxybuttersäurebildung nicht erklärlich.

Es stehen diese Resultate in Widerspruch mit Durchblutungsversuchen Embdens ${ }^{1}$ ) der bei Verwendung von 1-Leucin Acetonbildung nicht beobachtete, wohl dagegen bei Durchblutung mit d-Leucin. Wir haben deshalb die Versuche Embdens wiederholt. (Vgl. folgenden Abschnitt).

Da nun durch diese Untersuchungen zunächst die Frage der Bildung von Oxybuttersäure beim Diabetiker aus der von uns bearbeiteten Gruppe von Substanzen abgeschlossen erscheint, geben wir zum Schluß eine Übersicht unserer Resultate der verschiedenen Arbeiten in Kurvenform. Dieselben erscheinen uns deshalb wertvoll, weil sie einen Vergleich der Wirkung dieser Substanzen auf die Oxybuttersäurebildung in den verschiedenen Stadien des Diabetes, zum Teil bei demselben Patienten, ermöglichen. Die verabreichte Kost ist zur linken Seite der Kurven angegeben.

Durehblutungsversuche mit d- und l-Leucin.

Im Anschluß an den Befund der verschiedenen Wirkungen des d. und l-Leucin auf die Oxybuttersäureausscheidung, haben wir das Verhalten dieser Substanzen bei der Durchblutung der Hundeleber untersucht. Bei ähnlichen Versuchen konnte Embden ${ }^{2}$ ) feststellen, daß das synthetische $d-1$-Leucin und $d$-Leucin in beträchtlichem Maße Aceton bildeten, während das mit dem Eiweißleucin identische 1-Leucin kein Aceton lieferte. Wir benutzten zu unseren Versuchen dieselben Präparate, die dem Diabetiker gegeben worden waren. In einzelnen Versuchen wandten wir auch Eiweißleucin an, das wir aus Casein dargestellt und annähernd von Isoleucin befreit hatten (nach dem Vorschlage von F. Ehrlich). Leucin, in das Kupfersalz verwandelt, wurde mit Methylalkohol solange extrahiert, bis sich nichts mehr löste, das Kupfersalz wurde dann mit $\mathrm{H}_{2} \mathrm{~S}$ zersetzt und das Leucin durch Umkristallieren gereinigt.

1) Embden. Über das Verhalten der optisch isomeren-Leucine in der Leber. Hofmeisters Beiträge XI 348 (1908).

2) Embden. loc. cit. 
Kurve 6 (Tabelle II dieser Arbeit).

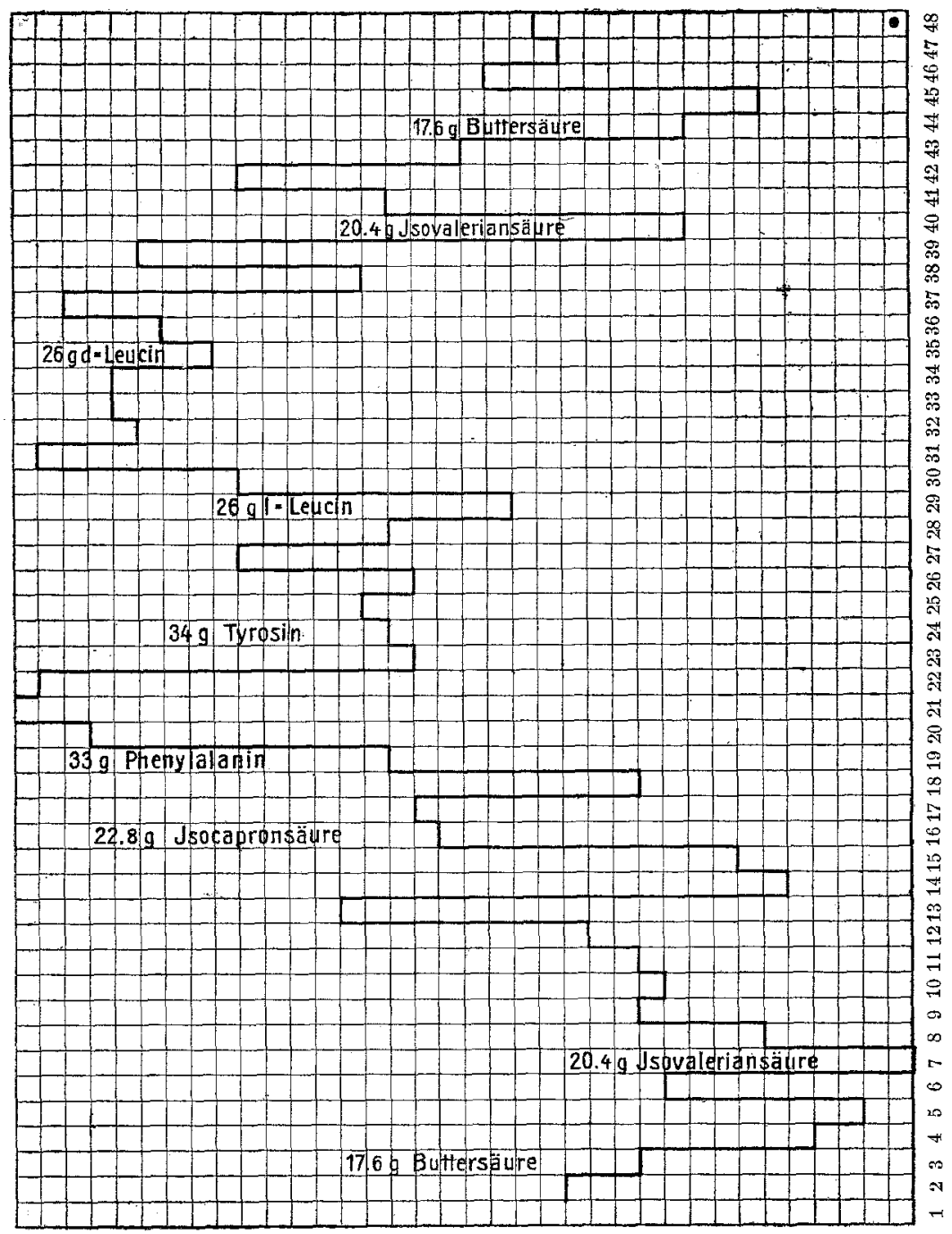

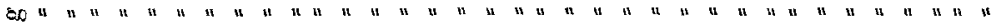

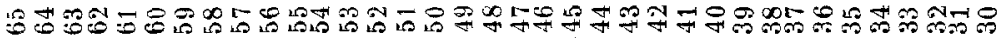

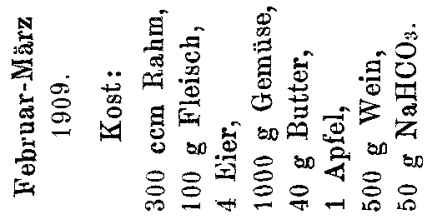


138 VII. BAER u. BrCM: Über den Abbau von Fettsăuren beim Diabetes melitus.

Tabelle III.

Versuche mit l-Leucin.

\begin{tabular}{|c|c|c|c|c|c|}
\hline 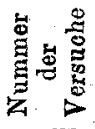 & $\begin{array}{l}\text { Dem Duroh- } \\
\text { blutungsblute } \\
\text { zugesetzte } \\
\text { Substanz }\end{array}$ & $\begin{array}{l}\text { Dauer der } \\
\text { Durch- } \\
\text { blutung }\end{array}$ & $\begin{array}{l}\text { Leber- } \\
\text { gewicht }\end{array}$ & $\begin{array}{c}\text { Im ganzen ge- } \\
\text { bildete Menge } \\
\text { Acetonin } \\
\text { in } 1000 \mathrm{cb} \mathrm{m}\end{array}$ & Bemerkungen \\
\hline 1. & $\begin{array}{l}1.7 \mathrm{~g} \text { l-Leucin } \\
\text { synthetisch }\end{array}$ & 65 Min. & $270 \mathrm{~g}$ & $38.4 \mathrm{mg}$ & gute Durohblut. \\
\hline $\begin{array}{l}2 \\
3\end{array}$ & $\begin{array}{c}1.7 \mathrm{~g}= \\
1.7 \mathrm{~g} \text { Eiweiß- } \\
\text { lenein }\end{array}$ & $\begin{array}{l}61 \text { Min. } \\
70 \text { Min. }\end{array}$ & $\begin{array}{l}255 \mathrm{~g} \\
270 \mathrm{~g}\end{array}$ & $\begin{array}{l}56.4 \mathrm{mg} \\
48 \mathrm{mg}\end{array}$ & $\begin{array}{l}\text { gute Durchblut. } \\
\text { gute Durchblut. }\end{array}$ \\
\hline 4 & $\left.1.7 \mathrm{~g} \begin{array}{c}1.0 \mathrm{~g} \text { Eiwei } \beta \\
0.7 \mathrm{~g} \text { synthet. }\end{array}\right\} 1$. & 70 Min. & $270 \mathrm{~g}$ & $54 \mathrm{mg}$ & $\begin{array}{l}\text { ziemlich gute } \\
\text { Durchblutung }\end{array}$ \\
\hline
\end{tabular}

Versuche mit d-Leucin.

\begin{tabular}{l|l|l|l|l|l}
1 & $1.7 \mathrm{~g}$ d-Leucin & $60 \mathrm{Min}$. & $230 \mathrm{~g}$ & $60.6 \mathrm{mg}$ & sehr gute Durch- \\
blutung \\
2 & $1.7 \mathrm{~g}$ d-Leucin & $68 \mathrm{Min}$. & $363 \mathrm{~g}$ & $53.4 \mathrm{mg}$ & $\begin{array}{l}\text { gute Durchblut. } \\
\text { mäB. Durchblut. }\end{array}$
\end{tabular}

Wie aus den Zahlen hervorgeht, zeigen unsere Versuche keinen deutlichen Unterschied zwischen d- und l-Leucin, Die geringe Differenz, die in einzelnen Versuchen zugunsten des $d$-Leucins besteht, kann auf Zufälligkeiten (Größe der Leber, Art der Durchblutung) beruhen. Die Versuche an der Hundeleber bieten demnach keine Erklårung für: das verschiedene Verhalten der beiden Substanzen im mensohlichen Organismus. 